\title{
"The only place I know that I can send patients to": Chicago-area generalist providers' perceptions of Planned Parenthood
}

\author{
Samuel R Mendez ${ }^{1}$, Allison Linton ${ }^{1}$, Laura Tom ${ }^{1}$ and Melissa Simon ${ }^{1-3 *}$ \\ ${ }^{1}$ Department of Obstetrics and Gynecology, Northwestern University Feinberg School of Medicine, USA \\ ${ }^{2}$ Department of Preventive Medicine and Medical Social Sciences, Northwestern University Feinberg School of Medicine, USA \\ ${ }^{3}$ Robert H Lurie Comprehensive Cancer Center, USA
}

\begin{abstract}
Context: This qualitative study explores generalist health care providers' perceptions of Planned Parenthood in relation to their own practice in an integrated health care system. This study addresses a gap in the literature around perceptions of Planned Parenthood among health care providers in the increasingly common context of integrated health care systems.
\end{abstract}

Methods: Providers were recruited at 4 hospitals in a Chicago-area integrated health care system. Semi-structured interviews were conducted with 37 generalist providers. Interview transcripts were analyzed with an inductive, constant comparative approach.

Results: We found key themes around providers' perceptions of Planned Parenthood in their abortion referral process: perceptions of Planned Parenthood as a uniform, collective abortion provider; perceptions of Planned Parenthood as a recognizable name associated with abortion; working around provider-level knowledge barriers; working around patient-level cost barriers; minimally facilitated referrals; and referrals to unnamed/unknown providers.

Conclusions: Our findings shed light on Planned Parenthood clinics' unique role in family planning referrals. While Planned Parenthood clinic sites differ in their availability of services, most providers focused on Planned Parenthood overall as a fallback option for abortion referrals. These findings suggest the need to empower generalist providers with comprehensive information about local family planning resources. These findings also highlight an opportunity for integrated health systems to work more closely with Planned Parenthood locations their providers may refer to.

\section{Introduction}

Planned Parenthood clinics offer a breadth of services including cancer screenings, vaccinations, and contraception services. Planned Parenthood clinics' notable role in women's health care has been documented, as in the case of interruptions to contraception usage observed after Planned Parenthood clinics were excluded from a Texas family planning program $[1,2]$. This role is also visible in associations between geographic access to Planned Parenthood clinics and lower female high school dropout rates, compared with geographic access to Title X clinics overall [3].

Despite this broader impact, Planned Parenthood has an overall association with abortion amidst political debates about "defunding" the organization [4]. Although Planned Parenthood is a significant abortion provider across the United States, there is considerable variation in abortion services offered at individual clinics. For example, out of the 10 Chicago-area Planned Parenthood locations, 6 clinics offer medication abortion up to 10 weeks gestation, and only 3 offer surgical abortion services, up to 19 weeks and 6 days gestation [5].Such clinic-level variation is important in the context of referrals, in which a particular site offers a limited range of services that cannot meet every single patient's needs.

Existing literature suggests that knowledge and training [6,7], as well as personal beliefs and professional obligation can impact health care providers' abortion referral practices [8,9]. Research suggests that family practice and obstetrics and gynecology providers may provide indirect abortion referrals [10], with some findings suggesting that such referrals from obstetrics and gynecology providers may include directing patients to a Planned Parenthood website [11]. However, generalist providers' views of Planned Parenthood in the context of abortion referrals are largely unknown, especially within integrated academic health care systems. These providers' views of Planned Parenthood are important because of the formal role that primary care providers play as health care gatekeepers for many patients [12], as well as their potential role as trusted sources of family planning information for traditionally underserved patients [13]. The context of integrated academic health care systems is important as there is increasing vertical integration of physician practices, and many regions' largest integrated systems contain an academic medical institution [14]. As more patients

*Correspondence to: Melissa A Simon, Departments of Obstetrics \& Gynecology, Northwestern University Feinberg School of Medicine, 633 N. St Clair, Suite 1800, Chicago, IL 60611, USA, Tel: (312) 503-8780, Fax: (312) 5035858, E-mail: m-simon2@northwestern.edu

Key words: abortion, public health, qualitative research methods, service providers, United States

Received: August 23, 2018; Accepted: September 17, 2018; Published: September 20, 2018 
receive primary and reproductive health care in these systems, it becomes more important to understand how providers in these systems fit into a broader family planning context. Therefore, this study seeks to explore generalist providers' perceptions of Planned Parenthood and its relationship to their practice within an integrated system.

\section{Methodology}

\section{Setting}

This study was conducted at a Chicago-area integrated health care system comprising a primary urban academic hospital and several suburban affiliate hospitals. Hospital integration took place on a systemby-system basis starting in 2010. This study focused on the primary urban academic center and the 3 community-based suburban facilities that finalized integration before 2015, in time for consideration in our study design.

The primary academic institution has a family planning and contraception subspecialty program that provides abortion services through 24 weeks of pregnancy: first trimester medication abortion, first trimester surgical abortion, second trimester dilation and evacuation, and second trimester induction of labor. Referrals to these subspecialty services are available to patients served throughout the integrated health care system. However, providers across the integrated system may refer patients to any other service providers they are aware of. There are no system restrictions on where they may refer. Tables 1 and 2 present the insurance coverage mix of each hospital in this study [15]. At the time of data collection, the Illinois Department of Healthcare and Family Services classified abortion as a non-covered service in its guidelines for fee-for service Medicaid providers, except when necessary to preserve the life or health of the mother, or in cases of rape or incest [16]. Private insurance coverage of abortion services varies by individual insurance plan.

No formal referral relationship exists between the integrated health system and Planned Parenthood affiliate clinics. Planned Parenthood locations are among the multiple options available to providers making external abortion referrals in the greater Chicago area. These external sites vary in geographic location, services offered, wait times, cost, and accepted insurance. Among Chicago-area Planned Parenthood locations, 1 suburban location and 2 Chicago locations offer surgical in-clinic abortions up to 19 weeks and 6 days gestation. One suburban location and 5 Chicago locations offer medication abortion up to 10 weeks and 0 days gestation [5].

Finally, Illinois is a state with relatively few legal regulations on abortion: there is no required counseling before receiving an abortion; no mandated waiting period; no limits on private insurance abortion coverage; and no parental consent required for adolescents [17].

\section{Sample and recruitment}

We recruited a convenience sample of general health care providers via departmental and division e-mail listservs from the fields of primary care, general internal medicine, general pediatrics, and general obstetrics and gynecology. Generalist health care providers (Medical Doctor, Osteopathic Doctor, Nurse Practitioner, Advanced Practice Nurse, Certified Nurse-Midwife, and Physician Assistant) practicing in these fields were invited to participate via this general e-mail method. Providers were excluded if they received subspecialty training beyond residency, were not affiliated with one of hospitals in this study, or if reproductive age women made up less than $25 \%$ of self-reported patient population.

Study participation was described to providers as an in-person interview lasting up to an hour focusing on three topics: comfort and willingness to provide family planning and abortion referrals; referral sites; and perceived barriers to providing family planning and abortion referrals. Additional recruitment was done in person with print versions of the recruitment script in the obstetrics-gynecology department at the primary academic location. Northwestern University's Institutional Review Board approved this study. Informed consent was obtained in person from each participant immediately before each interview.

\section{Data Collection}

Two authors conducted semi-structured in-person interviews between July 2016 and January 2017. Our qualitative instrument included questions about practice demographics, and the majority of questions focused on current practices around family planning referrals. The qualitative instrument is presented in Table 3. A literature review of medical provider practices and perceptions around family planning referrals was conducted to inform the interview questions. The interviews were audio recorded and transcribed verbatim. Field notes were documented after each interview. Providers received a US\$50 gift card for their participation.

\section{Analysis}

Field notes, memos, and transcripts were managed with ATLAS. ti. The codebook was further refined and additional codes identified at team meetings during the initial phase of the interview process. The final analysis used 84 codes to classify qualitative data across 7 distinct topic areas: (1) contraception service referral patterns, (2) prenatal service referral patterns, (3) discussion of pregnancy options, (4) personal-level abortion referral barriers, (5) system-level abortion referral barriers, (6) societal-level abortion referral barriers, and (7) scope of professional responsibility in abortion referrals. Two authors (AL, SM) used the codebook to identify trends in qualitative data around family planning referral practices. To ensure more than $80 \%$

Table 1. Inpatients Served by Source, Calendar Year 2015 [15]

\begin{tabular}{|c|c|c|c|c|c|c|}
\hline Hospital & Medicare (\%) & Medicaid (\%) & Other Public (\%) & Private Insurance (\%) & Private Pay (\%) & Charity Care (\%) \\
\hline Downtown Academic Center & 28.9 & 15.3 & 0.1 & 53.2 & 1.1 & 1.4 \\
\hline Suburban Community Hospital A & 38.6 & 6.6 & 3.1 & 45.8 & 4.0 & 2.0 \\
\hline Suburban Community Hospital B & 48.6 & 9.4 & 0.3 & 39.9 & 0.4 & 1.4 \\
\hline Suburban Community Hospital C & 36.1 & 15.3 & 0.1 & 45.7 & 0.4 & 2.5 \\
\hline
\end{tabular}

Table 2. Outpatients Served by Source, Calendar Year 2015 [15]

\begin{tabular}{|c|c|c|c|c|c|c|}
\hline Hospital & Medicare (\%) & Medicaid (\%) & Other Public (\%) & Private Insurance (\%) & Private Pay (\%) & Charity Care (\%) \\
\hline Downtown Academic Center & 37.4 & 9.9 & 0.1 & 49.2 & 2.2 & 1.3 \\
\hline Suburban Community Hospital A & 26.5 & 9.3 & 1.3 & 58.8 & 3.0 & 1.1 \\
\hline Suburban Community Hospital B & 31.5 & 11.7 & 0.1 & 54.6 & 0.7 & 1.3 \\
\hline Suburban Community Hospital C & 26.7 & 13.0 & 0.2 & 58.2 & 0.9 & 1.0 \\
\hline
\end{tabular}


Table 3. Qualitative instrument (semi-structured interview guide) ${ }^{\mathrm{a}}$

Discussion of options

- Walk me through what a first visit after a positive pregnancy test looks like. What do you discuss? (OR: If a patient is diagnosed with a new pregnancy in your practice, what type of care does she receive?)

o Do you provide pregnancy options counseling? Why/why not?

o If not, do you refer her to someone for options counseling?

o Are patients asked about whether they want to keep their pregnancy before initiating/being referred to prenatal care? (Non-OB/GYN specific)

- Tell me about what care a woman receives if she wants to continue the pregnancy.

o If she is referred for prenatal care, where do you refer?

Barriers for referral

- If you learn that a patient is unsure about continuing a pregnancy, walk me through what that conversation would look like. (OR: Tell me about what care she would receive if she is unsure about continuing the pregnancy.)

- How do you discuss this with her?

- Do you refer her to someone to discuss this?

- If you don't refer, how do you discuss this with her?

- If a patient tells you she wants an abortion, what would that conversation look like? (OR: Tell me about what care she would receive if she wants an abortion.)

- How do you discuss this with her?

- Do you refer her to someone to discuss this?

- Do you refer her to someone who can perform the abortion?

- Tell me about what things make referring for abortion difficult for you.

- Are there emotional barriers?

- System barriers?

- Other barriers?

- What do you think could be done to make abortion referrals easier for you?

- Alternate sources of support, office protocols, etc.

- Systemic changes (EMR, 24hr phone line, etc.)

\section{Referral patterns}

- How has your referral process for specialty services changed since the [integrated system] merger?

- Which specialty services have changed?

- Which are the same as before the merger?

- (For [suburban location] practitioners) Has the merger made you more apt to refer patients to subspecialty care within [the integrated system]? Why or why not?

- What factors do you consider as you decide where to refer a patient for any specialty services?

-What are some of the obstacles you encounter when referring patients?

- What would be helpful in supporting you to refer a woman to subspecialty care of any kind?

- What factors do you consider as you decide where to refer a patient for pregnancy care?

- How does your decision process change or differ for patients who desire abortion?

- Has your referral process changed for abortion services since the [integrated system] merger? Why or why not?

- Has the merger made you more likely to refer women to the Family Planning service at [the primary academic hospital]?

aAs this qualitative study used semi-structured interview methods, questions were not necessarily asked in this order or with this exact phrasing, allowing the participants' responses to shape the interview.

consensus, inter-coder reliability was assessed after the first 10 coded interviews.

An inductive thematic analysis was performed [18], elucidating key themes around Planned Parenthood in relation to the patterns observed from coding the interviews. A constant comparative analysis method was used to generate a theoretical understanding of phenomena we observed in participant descriptions of Planned Parenthood's role in their family planning referrals, within and between participant strata [19]. Analysis was performed until thematic saturation was achieved within each stratum for themes regarding barriers, knowledge, and perceptions of abortion referral processes.

\section{Results}

Interviews were conducted with 37 providers: 14 in obstetrics and gynecology, 10 in internal medicine, 8 in pediatrics, and 5 in family medicine. Most participants were in practice, affiliated with their respective institution prior to integration, or were affiliated with the primary academic institution prior to integration of 2 suburban hospital systems. One participant was a resident at a hospital of interest prior to their practice affiliation there, and 6 participants became affiliated with their respective institutions in the system after integrations were finalized. Table 4 provides a summary of participant characteristics.

Interviews ranged from approximately 8 to 26 minutes in length, with a mean length of 15 minutes. Without any direct mention of Planned Parenthood, most providers brought up Planned Parenthood in discussions of their referral patterns pregnancy options counseling
Table 4. Demographic characteristics of participants $(\mathrm{N}=37)$

\begin{tabular}{|l|c|}
\hline Demographic Characteristics & Value \\
\hline Gender, $\mathbf{n}(\%)^{\mathbf{a}}$ & $35(95)$ \\
\hline Female & $2(5)$ \\
\hline Male & \\
\hline Clinician type, $\mathbf{n} \mathbf{( \% )}^{\mathbf{a}}$ & $33(89)$ \\
\hline MD, DO & \\
\hline NP, APN, CNM, PA & $4(11)$ \\
\hline Specialty by Location, $\mathbf{n}(\mathbf{\%})^{\mathbf{a}}$ & \\
\hline Family Medicine, Community-based/Suburban & $5(14)$ \\
\hline General Internal Medicine, Community-based/Suburban & $4(11)$ \\
\hline General Internal Medicine, Primary Academic/Urban & $6(16)$ \\
\hline General Obstetrics and Gynecology, Community-based/Suburban & $5(14)$ \\
\hline General Obstetrics and Gynecology, Primary Academic/Urban & $9(24)$ \\
\hline General Pediatrics, Community-based/Suburban & $3(8)$ \\
\hline General Pediatrics, Primary Academic/Urban & $5(14)$ \\
\hline Obstetrics services offered in practice, $\mathbf{n}(\mathbf{\%})^{\mathbf{d}}$ & $12(86)$ \\
\hline General Obstetrics and Gynecology & $1(20)$ \\
\hline Family Medicine & $11.3(8.1)$ \\
\hline Years in practice, mean (SD) & \\
\hline
\end{tabular}

apercentages calculated as a portion of the total study population $(\mathrm{N}=37)$. ${ }^{b}$ Abbreviations used: Medical Doctor (MD), Osteopathic Doctor (DO).

${ }^{c}$ Abbreviations used: Nurse Practitioner (NP), Advanced Practice Nurse (APN), Certified Nurse-Midwife (CNM), and Physician Assistant (PA).

dPercentages calculated as a portion of the total participants in each specialty subgroup. 
and abortion. Follow-up questions and further probes from our qualitative instrument about decision-making and referral processes led us to identify 6 key themes about the providers' perceptions of Planned Parenthood in family planning referral processes. Two themes relate to the overall nature of abortion referrals to Planned Parenthood: "Minimally Facilitated Referrals" and "Referrals to Unnamed Providers". Two themes focus on descriptions of Planned Parenthood's relationship to abortion referral barriers: "Provider-Level Informational Barriers" and "Patient-Level Cost Barriers." Finally, two themes focus on perceptions specific to Planned Parenthood in the abortion referral decision-making process: "Uniform, Specialized Abortion Provider" and "Recognizable Name Associated with Abortion." We discuss these themes below and present exemplar quotations in table 5 .

\section{The nature of referrals to planned parenthood}

While 2 providers discussed Planned Parenthood referrals for contraception, most providers in our study only discussed Planned Parenthood in relation to abortion referral routes or pregnancy options discussions. Analysis of descriptions of these referrals led us to identify 2 key themes around providers' perceptions of their interactions with Planned Parenthood as a potential abortion referral site.

Minimally facilitated referrals: Many providers described solely verbal referrals to Planned Parenthood. Many described referral comprising publicly available information, such as one provider who described a process of "Googling 'Planned Parenthood' and printing it out." Many providers who described minimally facilitated referrals also described a lack of knowledge about abortion referrals overall.

Referrals to unnamed providers: Some providers described abortion referrals to a named provider within their practice or within the integrated system broadly. Apart from using an electronic referral system, providers described how they would also page or call an individual provider directly. In contrast, most providers only discussed the overarching name 'Planned Parenthood', without discussing a specific provider or clinic location for referrals. This finding was consistent among providers who also named individual physicians as abortion providers within the integrated system. Only 1 provider described referrals to providers at a Planned Parenthood clinic site by name.

Some described these referrals positively, as in the case of 1 provider discussing patients who wanted to "stay under the radar". Other providers expressed mixed feelings, such as an internal medicine provider who noted that some patients might be more comfortable receiving abortion care at a Planned Parenthood clinic, but that they would not "know the provider in the same kind of connected way".

\section{Planned parenthood and abortion referral barriers}

Most providers discussed Planned Parenthood in relation to abortion referral barriers. Participants either described how Planned Parenthood related to barriers while describing referral processes, or they elaborated on their perceptions of Planned Parenthood when asked about referral barriers.

Provider-Level informational barriers: Many providers discussed Planned Parenthood in relation to a lack of knowledge about abortion referral options. Most providers expressing uncertainty about abortion referral options still named Planned Parenthood as their intended referral site. Only 1 such provider mentioned that some Planned Parenthood clinics might not offer the necessary services. Providers who expressed little experience with undesired pregnancies also said they would provide a referral to Planned Parenthood. Notably, most providers who discussed Planned Parenthood in relation to a lack of knowledge of abortion referral sites did not discuss the integrated system's subspecialty family planning services.

Patient-Level cost barriers: Providers describing more knowledge of abortion services, especially obstetrics and gynecology providers, referred primarily to the integrated system's subspecialty family planning services. Planned Parenthood was described as an option to work around patient barriers, predominantly financial in nature. For example, one obstetrics and gynecology provider mentioned the integrated system's family planning subspecialty, Planned Parenthood, and another freestanding clinic as abortion provision sites that "can

Table 5. Exemplar quotations illustrating emergent themes

\section{Quotation}

Perceptions of Planned Parenthood as a Recognizable Name Associated with Abortion

Umm, my question would be like, I mean I can, I'm happy to personally provide a referral, but Planned Parenthood or something that had a little more of a reputation?

It's interesting. It's, it, there's not a lot of places around our office. And so, you know, everybody knows Planned Parenthood and so that's kind of what we end up doing.

And when you tell them Planned Parenthood, they kind of get this bad taste in their mouth.

'Perceptions of Planned Parenthood as a Uniform, Specialized Abortion Provider

So if someone needed an abortion, there was no choice to send them within the system. You had to find a Planned Parenthood. So I guess that's what my reflex is now.

Working Around Provider-Level Informational Barriers

See, I'm not familiar that there are any [obstetrics and gynecology] providers that would offer termination services? I'm actually not aware of any. So, therefore, pretty much that's the only place that I could think of would be Planned Parenthood.

I guess I would give them Planned Parenthood places. Because I don't even know how these guys...I guess I should ask the OBs how they would handle that, or who they suggest.

So unfortunately the only place I know that I can send patients to is Planned Parenthood.

Working Around Patient-Level Cost Barriers

'Cause what places will take — now Planned Parenthood I know is pretty, umm, pretty open to all insurances, but, some other places won't.

$\mathrm{Um}$, and then if someone does not have insurance coverage, we refer to Family Planning here, or Planned Parenthood, or [freestanding clinic]

because they can all do lower cost terminations than I can."

Referrals to Unnamed Providers at Planned Parenthood

If you go to Planned Parenthood, everyone's having the same kind of experience, but you won't know the provider in the same kind of connected way. Obstetrics and Gynecology

Minimally Facilitated Referrals to Planned Parenthood Clinics

Interviewer: And if you are giving that referral to Planned Parenthood, what does that referral look like?

Participant: It looks like me Googling Planned Parenthood and printing it out.

Participant Specialty

General Pediatrics

Internal Medicine

Obstetrics and Gynecology

General Pediatrics

Internal Medicine

General Pediatrics Family Medicine Internal Medicine

Obstetrics and Gynecology

Family Medicine 
all do lower cost terminations" than they could in their individual practice. Some providers described Planned Parenthood as the main option for patients with economic barriers to receiving abortion care in the integrated system, such as a participant who explained that Planned Parenthood is "pretty open to all insurances."

\section{Perceptions specific to planned parenthood in abortion referrals}

When discussing reasons behind their abortion referral routes, providers described Planned Parenthood as a uniform, specialized abortion provider with a recognizable name associated with abortion. Participants judged these factors both positively and negatively. These positive/negative judgments were described as factors in deciding whether a Planned Parenthood referral would be appropriate for specific patients.

Uniform, specialized abortion provider: Most providers made no mention of service variation between clinics or gestational age limits in a clinic's service offerings-factors that would impact which, if any, Planned Parenthood clinic a patient could feasibly be referred to. Few providers discussed differences in the availability of surgical versus medication abortion options, instead focusing on Planned Parenthood as a uniform abortion provider. This perceived narrow service offering was judged largely as a positive factor in referrals. For example, one provider explained that some patients might be more comfortable around others "having the same kind of experience" at Planned Parenthood, contrasting with the variety of patients and conditions treated in the integrated system. Others expressed confidence in the abortion care at Planned Parenthood, due to its perceived focus on abortion.

Recognizable name associated with abortion: Several providers discussed perceptions of Planned Parenthood's public recognition and association with abortion as a factor in their referrals. One provider reasoned, "...everybody knows Planned Parenthood and so that's kind of what we end up doing." Planned Parenthood's public image was judged both positively and negatively in the referral decision making process. On one hand, some providers cited Planned Parenthood's reputation as a reason to possibly refer there. Conversely, other providers described a negative perception of Planned Parenthood's reputation as a reason not to refer, like the obstetrician-gynecologist who explained that some patients "get a bad taste in their mouth" when they discuss Planned Parenthood.

Some providers described their own association of Planned Parenthood with abortion as a factor in their referrals. For example, one provider mentioned that their previous practice didn't provide abortion services, leading to a situation in which, "you had to find a Planned Parenthood. So I guess that's what my reflex is now."

\section{Discussion}

In this exploratory qualitative study, we conducted semi-structured interviews with health care providers in a Chicago-area integrated academic health care system. We found that providers perceived Planned Parenthood as a uniform, collective abortion provider with a widely recognizable name associated with abortion. Referrals to Planned Parenthood helped providers overcome barriers around lack of knowledge, as well as patients' financial barriers to receiving abortion services. These referrals were largely described as minimally facilitated and involving unnamed/unknown providers. Despite inclusion criteria covering reproductive age women in a provider's patient population, a small number of providers did not consider their individual scope of practice to include patients requiring an abortion referral. While this perspective was out of the scope of the 6 key themes, it still bears noting that these providers also named Planned Parenthood as a site that patients would either seek out on their own, or that a referral specialist in their practice would refer them to.

Considering the dearth of research on perceptions of Planned Parenthood across the broad scope of generalist health care providers, our findings make a useful contribution to women's health care research. This study highlights opportunities for important discussions around women's healthcare in integrated systems across the US. While this study focuses on just one integrated system, it spans a wide range of health care providers across specialties and geography, in an increasingly common type of system. This study presents findings from a range of participants reflecting the broad range of providers who provide reproductive health care integrated health care systems across the country.

Taken in context, in an integrated health care system with subspecialty family planning services, we interpret our findings as a sign of medical providers' perceptions of Planned Parenthood as a fallback abortion referral option, disconnected from the typical course of their primary care. Many providers expressed a lack of knowledge about abortion referral sites, with many seemingly unaware of the subspecialty family planning services available at the primary academic center. In these cases of lack of knowledge, Planned Parenthood seemed to serve as a fallback option when no others were perceived. It is unclear whether these providers would still consider Planned Parenthood a key referral option if they were better connected to family planning services within their own integrated system. However, our results from providers with more knowledge about abortion referrals still showed perceptions of Planned Parenthood as a fallback option of sorts. For these providers, their integrated system was the primary option, with Planned Parenthood named as the referral option in cases of patient financial barriers.

Across the broad range of providers, few discussed referrals to a specific Planned Parenthood location or the varying scope of abortion services between clinics. This clinic-by-clinic variation is important in a referral context, as not all local Planned Parenthood locations offer abortion services. There is additional service variation among the Planned Parenthood locations that do offer abortion services, as not all of these locations offer both medical and surgical options.

These findings point, in part, to success on the part of Planned Parenthood Federation of America as a recognizable, accessible family planning health services provider. No other abortion provision site was discussed as ubiquitously by providers in our study, not even the integrated system's family planning subspecialty. It is notable that providers who described uncertainty around abortion referrals still named Planned Parenthood as an option for their patients. These findings point to a unique, critical role for Planned Parenthood among generalist health care providers.

Additionally, we interpret our findings to highlight ways that providers, in positions of trust and authority, may be able further bolster Planned Parenthood's reputation as a safe, accessible source of medical care. While the providers in our study did not describe Planned Parenthood negatively, they did describe a hands-off approach to Planned Parenthood referrals that may still impact the organization's broader image. Providing indirect referrals and directing patients to unknown providers may communicate that Planned Parenthood clinics are distinct from other trusted health care teams that receive more facilitated referrals. These interactions between providers and 
Planned Parenthood are important in a national context with highprofile public discussion around "defunding" Planned Parenthood $[20,21]$. Such discourse has impacted the medical community, leading health care professionals to speak out against calls to "defund" the organization [22-26]. Beyond these public calls-to-action, providers could take a more hands-on approach to referrals, and health care systems could facilitate continuity of care in such referral interactions.

Our study also points to an opportunity to increase provider knowledge of local abortion referral options, including but not limited to Planned Parenthood. The Association of Reproductive Health Professionals has suggested that professionals across a health care team can be empowered to make more active referrals in pursuit of better health care [27]. Our own findings contribute to this understanding of abortion referral-making by highlighting the need to equip generalist providers with knowledge of local resources. It is beneficial that the "Planned Parenthood" name can serve as a starting point for providers who otherwise lack knowledge or experience around abortion referrals. However, patients could be made more vulnerable, and their health care options more limited, if their health care provider perceives only 1 organization as a referral option, in a context where resources for patient self-searching may yield mixed results [28]. Further, patients' quality of care could be diminished, especially if the services needed for a particular abortion do not align with a Planned Parenthood referral due to factors of gestational age, availability of medical/surgical abortion options, or geographic location.

Finally, this study raises key questions for integrated health care systems. If Planned Parenthood clinics are "defunded" or otherwise impeded from providing accessible services, patients may need to seek an alternate abortion referral from a generalist provider. Where will patients eventually end up receiving abortion services and how long will it take them to get there? How can integrated health care systems ensure that all of their providers are aware of the abortion services available to them? In a shifting policy climate around abortion, the answers to these questions are important in delivering effective, timely reproductive health care in the US.

\section{Limitations}

Key limitations of this study should be noted. Foremost, we interviewed a convenience sample of health care providers. Selfselection bias may limit the scope of our findings: most providers in our study cited pro-choice views or other personal factors in their consideration of abortion referrals. Additionally, this study was conducted in a metro area with multiple abortion provision sites, in a state with limited regulations on abortion. This study took place at a time when state policy prohibited Medicaid coverage of abortion. Application of our findings to settings with fewer referral options, more varied views on abortion referrals, or with stricter abortion regulation warrants further research. Further, the Illinois policy on Medicaid coverage of abortion has changed since the time of data collection [29], and thus provider perceptions of patients' financial barriers to receiving abortion care may have changed. Finally, interviews took place between July 2016 and January 2017. The political climate surrounding the 2016 presidential election may have impacted providers' perceptions of the name "Planned Parenthood".

It should be noted that many providers may not have discussed Planned Parenthood beyond abortion due to their integrated system's scope of services aligning with patient population's needs. All providers were asked about referral patterns for prenatal care and contraception, and 2 providers discussed referrals to Planned Parenthood for contraception services. However, this may be specific to certain patient populations or geographic areas served by the integrated health care system. Thus, while providers discussed Planned Parenthood as an abortion provider, we cannot say what provider views are of Planned Parenthood and its broader service offering.

\section{Directions for future research}

Our findings set the groundwork for future research to explore Planned Parenthood's role for patients seeking care in integrated health care systems. Because integrated health care systems can easily cover large geographic areas and a variety of community contexts, further research including geographic information system methods and economic analysis may be needed to understand system-level service relationships with Planned Parenthood. This study also points to the need to examine connections between provider knowledge and the route of abortion referrals. Such research may highlight opportunities for interventions focusing on provider knowledge. Additionally, health care systems may be able to support their providers in working more closely with providers at Planned Parenthood clinics they refer to; further research into the efficiency of such referrals may be warranted to help shape system interactions. Finally, we suggest future research examine interactions between provider and patient views of Planned Parenthood, a factor that providers considered in their decisionmaking process.

\section{Conclusion}

Our study highlights the need to increase knowledge about Planned Parenthood clinic services across the range of generalist providers who provide reproductive health care. Planned Parenthood clinics provide accessible, safe, legal abortion services alongside critical cancer screenings, vaccinations, and contraception services. There is an opportunity for a range of providers to work more closely with Planned Parenthood as a trusted, known health care provider, rather than a disconnected abortion referral option.

Our findings shed light on the need for family planning departments in integrated health care systems to support generalist providers with information about the breadth of services available within their system. Reproductive health care is within the scope of many generalist practices. Thus, the increasingly common integrated health care system is uniquely positioned to empower a broad range of providers across large geographic areas to improve care. By better linking health care providers to family planning services, such health care systems can provide timely abortion referrals to patients needing them under varying circumstances of geography, insurance, gestational age, and clinical point of entry.

\section{Authorship and Contributions}

Melissa Simon conceived and designed the study, and she substantially contributed to data interpretation and the drafting of the manuscript; Dr. Simon approved the version to be published, and agrees to act as a guarantor of the work. Samuel Mendez, Allison Linton, and Laura Tom each substantially contributed to data acquisition, analysis and interpretation and the drafting of the manuscript; in addition, each approved the version to be published and agree to act as a guarantor of the work.

\section{Funding Information}

This study was supported by funding from the Society of Family Planning (Grant Number: SFPRF16-9) (MAS), with no influence on the study design, data analysis, writing, or submission for publication. 


\section{Competing Interest}

The authors declare that they have no competing interests. Melissa Simon is a member of the United States Preventive Services Task Force (USPSTF). This article is the sole responsibility of the authors and does not necessarily represent the views and policies of the USPSTF.

\section{References}

1. Stevenson AJ, Flores-Vazquez IM, Allgeyer RL, Schenkkan P, Potter JE (2016) Effect of Removal of Planned Parenthood from the Texas Women's Health Program. N Engl J Med 374: 853-860. [Crossref]

2. Woo CJ, Alamgir H, Potter JE (2016) Women's experiences after Planned Parenthood's exclusion from a family planning program in Texas. Contraception 93: 298-302. [Crossref]

3. Hicks-Courant KMD, Schwartz ALP (2016) Local Access to Family Planning Services and Female High School Dropout Rates. Obstet Gynecol 127: 699-705. [Crossref]

4. McCarthy M (2015) Democrats block vote on bill to deny federal funds to Planned Parenthood. BMJ 351: h4236. [Crossref]

5. Planned Parenthood of Illinois (2018) Abortion Services. https://www. plannedparenthood.org/planned-parenthood-illinois/patient-resources/abortionservices.

6. Dodge LE, Haider S, Hacker MR (2013) Knowledge of state-level abortion laws and regulations among reproductive health care providers. Womens Health Issues 23: e281-e286. [Crossref]

7. Steinauer JE, DePineres T, Robert AM, Westfall J, Darney P (1997) Training family practice residents in abortion and other reproductive health care: a nationwide survey. Fam Plann Perspect 29: 222-227. [Crossref]

8. French V, Anthony R, Berrios S, Crockett L, Steinauer JE (2016) A sense of obligation: attitudes and referral practices for abortion services among women's health providers in a rural US state. Clin Obstet Gynecol Reprod Med 2: 204-210.

9. Westfall JM, Kallail KJ, Walling AD (1991) Abortion attitudes and practices of family and general practice physicians. J Fam Pract 33: 47-51. [Crossref]

10. Homaifar N, Freedman L, French V (2017) "She's on her own": a thematic analysis of clinicians' comments on abortion referral. Contraception 95: 470-476. [Crossref]

11. Desai S, Jones RK, Castle K (2018) Estimating abortion provision and abortion referrals among United States obstetrician-gynecologists in private practice. Contraception 97 297-302. [Crossref]

12. Fang H, Liu H, Rizzo JA (2009) Has the use of physician gatekeepers declined among HMOs? Evidence from the United States. Int J Health Care Finance Econ 9: 183-195. [Crossref]
13. Blackstock OJ, Mba-Jonas A, Sacajiu GM (2010) Family Planning Knowledge: The Role of Social Networks and Primary Care Providers as Information Sources for African American Women. Am J Sex Educ 5: 128-143.

14. Cutler DM, Scott Morton F (2013) Hospitals, market share, and consolidation.JAMA 310: 1964-1970. [Crossref]

15. Illinois Health Facilities and Services Review Board (2015) Individual Hospita Profiles by Hospital Name,2015. Illinois.gov. [https://www2.illinois.gov/sites/hfsrb/ InventoriesData/FacilityProfiles/Documents/2015\%20Hospital\%20Profile\%2011-1617.pdf]. Accessed May 01, 2018.

16. Illinois Department of Health and Family Services (2016) Handbook for Practitioners Rendering Medical Services Chapter A-200 Policy and Procedures for Medical Services. Section A-223.3.7. Illinois.gov. 2016. [https://www.illinois.gov/hfs/ sitecollectiondocuments/a200.pdf]

17. Guttmacher Institute (2017) An overview of abortion laws. 2017; https://www guttmacher.org/state-policy/explore/overview-abortion-laws.

18. Todd NJ, Jones SH, Lobban FA (2012) "Recovery" in bipolar disorder: how can service users be supported through a self-management intervention? A qualitative focus group study. J Ment Health 21: 114-126. [Crossref]

19. Boeije H (2002) A Purposeful Approach to the Constant Comparative Method in the Analysis of Qualitative Interviews. Qual Quant 36: 391-409.

20. Brandhorst JK, Jennings FJ (2016) Fighting for funding: Values advocacy and Planned Parenthood's right-to-life. Public Relat Rev 42: 723-733.

21. Han L, Han L, Darney B, Rodriguez MI (2017) Tweeting PP: an analysis of the 2015-2016 Planned Parenthood controversy on Twitter. Contraception 96: 388-394. [Crossref]

22. Rubin R (2018) A Day in the Life: Planned Parenthood Physician Provides Reproductive Health Care to the Underserved. JAMA 319: 1081-1083. [Crossref]

23. Silver D, Kapadia F (2017) Planned Parenthood Is Health Care, and Health Care Must Defend It: A Call to Action. Am J Public Health 107: 1040-1041. [Crossref]

24. Lawrence HC, Ness DL (2017) Planned Parenthood Provides Essential Services That Improve Women's Health. Ann Intern Med 166: 443-444. [Crossref]

25. Ciccariello C (2017) Defunding Planned Parenthood-The Stakes for America's Women. JAMA Intern Med 177: 307-308. [Crossref]

26. Adams Hillard PJ (2017) Adolescent Reproductive Health Without Planned Parenthood. J Pediatr Adolesc Gynecol 30: 445-446. [Crossref]

27. Zurek M, O'Donnell J, Hart R, Rogow D (2015) Referral-making in the current landscape of abortion access. Contraception 91: 1-5. [Crossref]

28. Dodge L, Phillip SJ, Neo D, Nippita S, Paul M, Hacker M. (2017) Just Google it quality of information available online for abortion self-referral. Contraception 96: 274.

29. An act concerning abortion (2017) Public Act 100-0538. Illinois $100^{\text {th }}$ General Assembly.

Copyright: (C2018 Mendez SR. This is an open-access article distributed under the terms of the Creative Commons Attribution License, which permits unrestricted use, distribution, and reproduction in any medium, provided the original author and source are credited. 